\section{US dilutes smallpox vaccine supplies}

The United States government is seeking ways to increase production of smallpox vaccine in the current climate of bioterrorism since stores are insufficient to vaccinate all Americans. It has put vaccine contracts out to tender and is testing dilutions of existing stocks to serve as a stopgap measure until enough fullstrength vaccine is avallable.

Wyeth Ayest manufactured the original vaccine, Dryvax, but its production stopped in 1983. The National Institute of Allergy and Infectious Diseases (NIAID) conducted a pilot study in the spring of 2000 to see whether the potency of the vaccine had diminished. Those results showed that vaccine potency fit the same curves that were published more than 30 years ago. "In other words, it was still quite potent," Carole Heilman, director of NIAID's division of microbiology and infectious diseases, told Nature Medicine.

NIAID has now commenced a large dilution study with 650 healthy volunteers never previously vaccinated against smallpox. "We are testing two dilutions, $1: 5$ and $1: 10$, against undiluted vaccine," says Heilman. "We are asking two questions: What percentage of those recelving diluted vaccines have a 'take?' and for those who do not have a 'take' the first time, would revaccination result in a 'take?" " By 'take,' researchers mean "the presence of the characteristic skin lesion at the site of inoculation," explains NIAID director Anthony Fauct.

Fauci says they will also be measuring antibody titers as "this cannot be a trial of 'efficacy' since you obviously cannot challenge the vaccinees. In essence, you are looking at surrogate markers of protection." The dilution factors were chosen to allow for the greatest number of people to be vaccinated. Previous study showed that $70 \%$ were successfully vaccinated at a 1:10 dilution. "We do not know if $1: 5$ will change that significantly or not," admits Heilman. But if it does, "then you've expanded 15.4 million doses to 75 million," says Fauci. Results are due by the end of the year. Smallpox immunity can be achieved within a matter of days, so this could be an important protective measure should there be a sudden emergence of smallpox cases.

NIAID and other companies are pursuing alternative routes. These include a modified vaccinia strain that has much lower levels of replication, DNA vaccines expected in the Spring. and inactivated vaccines in combination with specialized adjuvants.

The government has not yet announced which companies will receive contracts to expand the smallpox vaccine supply, but Acambis, a UK company, was asked last year to supply a certain number of doses (Nature Med. 7, 1167; 2001). And although licensing a vaccine typically takes years, the possibility of using vaccine under an Investigational New Drug application is being considered in the case of an emergency.

Marlene Cimons, Washington, DC

\title{
New class of HIV drugs shows promise
}

Data presented at the recent European Conference on Clinical Aspects and Treatment of HIV Infection in Athens holds promise that medications being developed to combat multi-drug resistant HIV are nearing the market. The most recent analyses show that around $25 \%$ of patients undergoing antiretroviral therapy have developed multi-drug resistant HIV, and that the overall prevalence of resistance is growing (Nature Med. 7, 1016; 2001).

Phase II trials of T-20, a fusion inhibitor developed by North Carolina-based biotech company, Trimeris, reduced viral load by at least 10 -fold in $56 \%$ of patients receiving treatment. Results from Phase III studies are

T-20 targets HIV as its membrane fuses with the membrane of a host cell-a critical

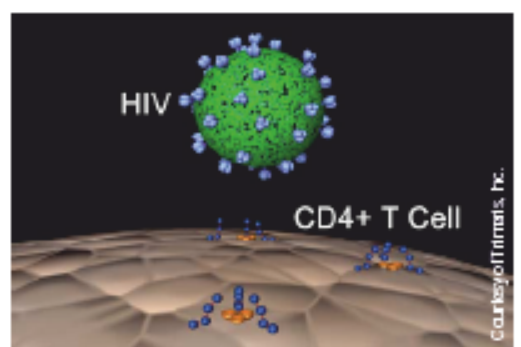

T-20 blocks HIV fusion with T-cells step in viral entry. Trimeris is developing a second-generation drug (T-1249) which is roughly two years behind T-20 in the development pipeline. Because T-20 and T-1249 are small peptides that target the viral protein gp41, the differences in their resistance profiles suggest that engineering changes to peptide drugs may provide a rapid means of responding to new waves of viral resistance. In addition, preliminary evidence suggests that fusion inhibitors and other inhibitors of viral entry, such as compounds that block virus-receptor interactions, may act synergistically.

Alan Dove, Philadelphia

\section{Rapid anthrax test developed}

A group at the Mayo Clinic has developed a rapid PCR-based test for detecting anthrax (Bacillus anthracis) in environmental as well as human swab samples. A posittve result can be obtained in less than 35 minutes-much faster than traditional microbiology techniques, which takes 24-48 hours.

Difficulties in identifying anthrax arise because of similarities of $B$. anthracis to other members of the $B$ cereus group, both visually and at the genomic level. Franklin Cockerill's group has taken advantage of the fact that virulent isolates of $B$. anthracis contain two plasmids in addition to their genomic DNA that have unique genes. They test for the $C a p B$ gene, which occurs on one of these plasmids.

The test is quick because it uses Roche's LightCycler machine, a microvolume fluorometer with an integrated thermal cycler. Very small samples are required-as little as one copy of template DNA. Analysis and quantification of the PCR products are made in the machine using fluorescence, avoiding the need to 'process' samples after the reaction is completed, thus saving an hour or more. Although some methods for breaking the anthrax spore coat (which is required when testing environmental samples) inhibit PCR, it is possible to disrupt the spore coat mechanically, which would$n$ 't affect the reaction.

Roche has scaled up production of the reagents required for the test and is in the process of distributing them to about 24 labs in the United States that have expressed interest and have the LightCycler machine. The test will be free until at least the end of the year and although Roche have offered the test to US federal departments, none have as yet taken up the offer.

The LightCycler protocol can also detect toxoplasmosis infection, and BCR-ABL mRNA translocation, which results in a large percentage of leukemias and is difficult to detect with traditional methods. Sarah Cooney, London 